\title{
Indestructibility, Instances of Strong Compactness, and Level by Level Inequivalence ${ }^{* \dagger}$
}

\author{
Arthur W. Apter $\$ \S$ \\ Department of Mathematics \\ Baruch College of CUNY \\ New York, New York 10010 USA \\ and \\ The CUNY Graduate Center, Mathematics \\ 365 Fifth Avenue \\ New York, New York 10016 USA \\ http://faculty.baruch.cuny.edu/apter \\ awapter@alum.mit.edu
}

November 24, 2008

(revised July 2, 2010)

\begin{abstract}
Suppose $\lambda>\kappa$ is measurable. We show that if $\kappa$ is either indestructibly supercompact or indestructibly strong, then $A=\left\{\delta<\kappa \mid \delta\right.$ is measurable, yet $\delta$ is neither $\delta^{+}$strongly compact nor a limit of measurable cardinals $\}$ must be unbounded in $\kappa$. The large cardinal hypothesis on $\lambda$ is necessary, as we further demonstrate by constructing via forcing two models in which $A=\emptyset$. The first of these contains a supercompact cardinal $\kappa$ and is such that no cardinal $\delta>\kappa$ is measurable, $\kappa$ 's supercompactness is indestructible under $\kappa$-directed closed, $\left(\kappa^{+}, \infty\right)$ distributive forcing, and every measurable cardinal $\delta<\kappa$ is $\delta^{+}$strongly compact. The second of these contains a strong cardinal $\kappa$ and is such that no cardinal $\delta>\kappa$ is measurable, $\kappa$ 's strongness is indestructible under $<\kappa$-strategically closed, $\left(\kappa^{+}, \infty\right)$-distributive forcing, and level by level inequivalence between strong compactness and supercompactness holds. The model from the first of our forcing constructions is used to show that it is consistent, relative to a supercompact cardinal, for the least cardinal $\kappa$ which is both strong and has its strongness indestructible under $\kappa$-directed closed, $\left(\kappa^{+}, \infty\right)$-distributive forcing to be the same as the least supercompact cardinal, which has its supercompactness indestructible under
\end{abstract}

*2010 Mathematics Subject Classifications: 03E35, 03E55.

${ }^{\dagger}$ Keywords: Supercompact cardinal, strongly compact cardinal, strong cardinal, indestructibility, non-reflecting stationary set of ordinals, level by level inequivalence between strong compactness and supercompactness.

¥The author's research was partially supported by PSC-CUNY grants and CUNY Collaborative Incentive grants.

$\S$ The author wishes to thank the referee for helpful comments, suggestions, and corrections which have been incorporated into the current version of the paper. 
$\kappa$-directed closed, $\left(\kappa^{+}, \infty\right)$-distributive forcing. It further follows as a corollary of the first of our forcing constructions that it is possible to build a model containing a supercompact cardinal $\kappa$ in which no cardinal $\delta>\kappa$ is measurable, $\kappa$ is indestructibly supercompact, and every measurable cardinal $\delta<\kappa$ which is not a limit of measurable cardinals is $\delta^{+}$strongly compact.

\section{Introduction and Preliminaries}

We begin with some key definitions. For any non-supercompact measurable cardinal $\delta$, let $\theta_{\delta}$ be the smallest cardinal greater than $\delta$ such that $\delta$ is not $\theta_{\delta}$ supercompact. Suppose now that $V$ is a model of ZFC in which if $\delta$ is measurable but not supercompact, then $\delta$ is $\theta_{\delta}$ strongly compact. $V$ is said to witness level by level inequivalence between strong compactness and supercompactness. The cardinal $\kappa$ is indestructibly supercompact if $\kappa$ 's supercompactness is preserved after forcing with a $\kappa$ directed closed partial ordering. The cardinal $\kappa$ is indestructibly strong if $\kappa$ 's strongness is preserved after forcing with a $<\kappa$-strategically closed, $(\kappa, \infty)$-distributive partial ordering. The degrees of indestructibility just mentioned are essentially standard (see, e.g., [16, 14, 11]) for supercompactness and strongness. ${ }^{1}$

It is a remarkable fact that the structure of the universe above a strong or supercompact cardinal $\kappa$ with suitable indestructibility properties can affect what happens at large cardinals below $\kappa$, assuming the universe is sufficiently rich. On the other hand, these effects can be mitigated if the universe contains relatively few large cardinals. For instance, in [8, Theorems 5 and 6$]$, it was shown that if $\kappa<\lambda$ are such that $\kappa$ is either indestructibly supercompact or indestructibly strong and $\lambda$ is $2^{\lambda}$ supercompact, then $B=\left\{\delta<\kappa \mid \delta\right.$ is $\delta^{+}$strongly compact yet $\delta$ is not $\delta^{+}$ supercompact\} must be unbounded in $\kappa$. However, if the universe contains a fairly small number of large cardinals, $\kappa$ is either strong or supercompact, and $\kappa$ 's supercompactness or strongness exhibits enough indestructibility, then $[8$, Theorem 8$]$ and $[4$, Theorem 1] indicate that $B$ may be empty. This sort of occurrence has been further examined in $[1,2,3]$.

The purpose of this paper is to continue studying this phenomenon, but in the context of

\footnotetext{
${ }^{1}$ For a strong cardinal $\kappa,[14$, Theorem 4.10] and the work of [11, Section 2] force indestructibility under $\kappa$ strategically closed partial orderings. However, as our proof of Lemma 3.4 will show, the techniques of [14, Theorem $4.10]$ actually force indestructibility under $<\kappa$-strategically closed, $(\kappa, \infty)$-distributive partial orderings.
} 
investigating the degree of strong compactness each measurable cardinal can manifest in universes containing either a supercompact or a strong cardinal with various indestructibility properties. Specifically, we prove the following theorems.

Theorem 1 Suppose $\lambda>\kappa$ is measurable and $\kappa$ is either indestructibly supercompact or indestructibly strong. Then $A=\left\{\delta<\kappa \mid \delta\right.$ is measurable, yet $\delta$ is neither $\delta^{+}$strongly compact nor a limit of measurable cardinals\} is unbounded in $\kappa$.

Theorem 2 Suppose $V \vDash " Z F C+\kappa$ is supercompact + No cardinal $\delta>\kappa$ is measurable". There is then a partial ordering $\mathbb{P} \subseteq V$ such that $V^{\mathbb{P}} \vDash " Z F C+\kappa$ is supercompact + No cardinal $\delta>\kappa$ is measurable". In addition, the following hold in $V^{\mathbb{P}}$ :

1. $\kappa$ 's supercompactness is indestructible under $\kappa$-directed closed, $\left(\kappa^{+}, \infty\right)$-distributive forcing.

2. $\kappa$ is the least strongly compact cardinal.

3. Every measurable cardinal $\delta<\kappa$ is $\delta^{+}$strongly compact.

Theorem 3 Suppose $V \vDash " Z F C+G C H+\kappa$ is the least cardinal which is both strong and $\kappa^{+}$ supercompact + No cardinal $\delta>\kappa$ is measurable". There is then a partial ordering $\mathbb{P} \in V$ such that $V^{\mathbb{P}} \vDash " Z F C+\kappa$ is strong + No cardinal $\delta>\kappa$ is measurable". In addition, the following hold in $V^{\mathbb{P}}$ :

1. $\kappa$ 's strongness is indestructible under $<\kappa$-strategically closed, $\left(\kappa^{+}, \infty\right)$-distributive forcing.

2. $\kappa$ is the least strong cardinal.

3. Level by level inequivalence between strong compactness and supercompactness holds.

As a corollary to Theorem 2 and its proof, we have the following two theorems.

Theorem 4 It is consistent, relative to the existence of a supercompact cardinal, for the least strong cardinal $\kappa$ whose strongness is indestructible under $\kappa$-directed closed, $\left(\kappa^{+}, \infty\right)$-distributive forcing to be the same as the least supercompact cardinal, which has its supercompactness indestructible under $\kappa$-directed closed, $\left(\kappa^{+}, \infty\right)$-distributive forcing. 
Theorem 5 Suppose $V \vDash " Z F C+\kappa$ is supercompact + No cardinal $\delta>\kappa$ is measurable". There is then a partial ordering $\mathbb{P} \subseteq V$ such that $V^{\mathbb{P}} \vDash " Z F C+\kappa$ is supercompact + No cardinal $\delta>\kappa$ is measurable". In addition, the following hold in $V^{\mathbb{P}}$ :

1. $\kappa$ is indestructibly supercompact.

2. $\kappa$ is the least strongly compact cardinal.

3. Every measurable cardinal $\delta<\kappa$ which is not a limit of measurable cardinals is $\delta^{+}$strongly compact.

We take this opportunity to make a few remarks concerning Theorems $1-5$. The limited amount of indestructibility forced in Theorems 2 and 3 is due to the necessity in our proofs of preserving a nontrivial degree of strong compactness. However, if we weaken the requirement in Theorem 2 of all measurable cardinals $\delta<\kappa$ being $\delta^{+}$strongly compact to only measurable cardinals $\delta<\kappa$ which are not themselves limits of measurable cardinals being $\delta^{+}$strongly compact, then it is possible for $\kappa$ to be a fully indestructible supercompact cardinal. Also, as our proof will show, the degrees of indestructibility mentioned in the statement of Theorem 1 can be weakened. In particular, $\kappa$ 's supercompactness can be indestructible under $\kappa$-directed closed, $\left(\kappa^{+}, \infty\right)$-distributive forcing, and $\kappa$ 's strongness can be indestructible under $<\kappa$-strategically closed, $\left(\kappa^{+}, \infty\right)$-distributive forcing. This provides a nice balance between Theorem 1 and Theorems 2 and 3. Further, note that the hypotheses used in Theorem 3 are much weaker than the existence of a supercompact cardinal, yet much stronger than the existence of a strong cardinal. The first of these facts is implied by [7, Lemma 2.1] and its proof, since if $\kappa$ is supercompact, then $\kappa$ is a limit of cardinals $\delta$ which are both $2^{\delta}$ supercompact and strong. The second of these facts is also implied by [7, Lemma 2.1], since if $\kappa$ is $2^{\kappa}$ supercompact and strong, then $\kappa$ is a limit of strong cardinals. In addition, the proof of [3, Theorem 3] provides the existence of a model in which the least strong cardinal $\kappa$ whose strongness is indestructible under $\kappa$-directed closed, $(\kappa, \infty)$-distributive forcing is the same as the least supercompact cardinal, which has its supercompactness indestructible under $\kappa$-directed 
closed, $(\kappa, \infty)$-distributive forcing. ${ }^{2}$ In this model, there are no Mahlo cardinals above $\kappa$. The model witnessing the conclusions of Theorem 4 will be the model of Theorem 2, and hence will be such that there are may be Mahlo cardinals, weakly compact cardinals, Ramsey cardinals, etc. above the supercompact cardinal $\kappa$. Thus, by slightly weakening the amount of indestructibility forced, it is possible to get a witnessing model with potentially more large cardinals above the supercompact cardinal in question.

We conclude Section 1 with a very brief discussion of some preliminary material. We presume a basic knowledge of large cardinals and forcing. A good reference in this regard is [15]. When forcing, $q \geq p$ means that $q$ is stronger than $p$. When $G$ is $V$-generic over $\mathbb{P}$, we abuse notation slightly and take both $V[G]$ and $V^{\mathbb{P}}$ as being the generic extension of $V$ by $\mathbb{P}$. We also abuse notation slightly by occasionally confusing terms with the sets they denote, especially for ground model sets and variants of the generic object. For $\alpha<\beta$ ordinals, $[\alpha, \beta],(\alpha, \beta],[\alpha, \beta)$, and $(\alpha, \beta)$ are as in standard interval notation. For $\kappa$ a measurable cardinal, the normal measure $\mathcal{U}$ over $\kappa$ has trivial Mitchell rank if for $j: V \rightarrow M$ the elementary embedding generated by $\mathcal{U}, M \vDash " \kappa$ is not measurable".

Suppose $\kappa$ is a regular cardinal. The partial ordering $\mathbb{P}$ is $\kappa$-directed closed if for every directed set $D$ of conditions of size less than $\kappa$, there is a condition in $\mathbb{P}$ extending each member of $D$. $\mathbb{P}$ is $\kappa$-strategically closed if in the two person game in which the players construct an increasing sequence $\left\langle p_{\alpha} \mid \alpha \leq \kappa\right\rangle$, where player I plays odd stages and player II plays even stages, player II has a strategy ensuring the game can always be continued. $\mathbb{P}$ is $\prec \kappa$-strategically closed if in the two person game in which the players construct an increasing sequence $\left\langle p_{\alpha} \mid \alpha<\kappa\right\rangle$, where player I plays odd stages and player II plays even stages, player II has a strategy ensuring the game can always be continued. $\mathbb{P}$ is $<\kappa$-strategically closed if $\mathbb{P}$ is $\delta$-strategically closed for every $\delta<\kappa$. $\mathbb{P}$ is $(\kappa, \infty)$-distributive if the intersection of $\kappa$ many dense open subsets of $\mathbb{P}$ is dense open. It therefore follows that any partial ordering $\mathbb{P}$ which is $\kappa$-directed closed is also $<\kappa$-strategically closed, and any partial ordering which is $\kappa$-strategically closed is $(\kappa, \infty)$-distributive. It further follows that

\footnotetext{
${ }^{2}$ Both this theorem and Theorem 4 show that with a certain amount of indestructibility, it is relatively consistent for the least strong cardinal to be the least supercompact cardinal. By [7, Lemma 2.1], this is certainly impossible without indestructibility added on.
} 
forcing with any partial ordering $\mathbb{P}$ which is $\left(\kappa^{+}, \infty\right)$-distributive preserves either the $\kappa^{+}$strong compactness or $\kappa^{+}$supercompactness of $\kappa$, since forcing with $\mathbb{P}$ adds no new subsets of $P_{\kappa}\left(\kappa^{+}\right)$.

A corollary of Hamkins' work on gap forcing found in $[12,13]$ will be employed in the proof of Theorems $2-5$. We therefore state as a separate theorem what is relevant for this paper, along with some associated terminology, quoting from $[12,13]$ when appropriate. Suppose $\mathbb{P}$ is a partial

ordering which can be written as $\mathbb{Q} * \dot{\mathbb{R}}$, where $|\mathbb{Q}|<\delta, \mathbb{Q}$ is nontrivial, and $\vdash_{\mathbb{Q}}$ " $\mathbb{R}$ is $\delta$-strategically closed". In Hamkins' terminology of $[12,13], \mathbb{P}$ admits a gap at $\delta$. In Hamkins' terminology of $[12,13], \mathbb{P}$ is mild with respect to a cardinal $\kappa$ iff every set of ordinals $x$ in $V^{\mathbb{P}}$ of size below $\kappa$ has a "nice" name $\tau$ in $V$ of size below $\kappa$, i.e., there is a set $y$ in $V,|y|<\kappa$, such that any ordinal forced by a condition in $\mathbb{P}$ to be in $\tau$ is an element of $y$. Also, as in the terminology of $[12,13]$ and elsewhere, an embedding $j: \bar{V} \rightarrow \bar{M}$ is amenable to $\bar{V}$ when $j \uparrow A \in \bar{V}$ for any $A \in \bar{V}$. The specific corollary of Hamkins' work from $[12,13]$ we will be using is then the following.

Theorem 6 (Hamkins) Suppose that $V[G]$ is a generic extension obtained by forcing with $\mathbb{P}$ that admits a gap at some regular $\delta<\kappa$. Suppose further that $j: V[G] \rightarrow M[j(G)]$ is an embedding with critical point $\kappa$ for which $M[j(G)] \subseteq V[G]$ and $M[j(G)]^{\delta} \subseteq M[j(G)]$ in $V[G]$. Then $M \subseteq V$; indeed, $M=V \cap M[j(G)]$. If the full embedding $j$ is amenable to $V[G]$, then the restricted embedding $j \uparrow V: V \rightarrow M$ is amenable to $V$. If $j$ is definable from parameters (such as a measure or extender) in $V[G]$, then the restricted embedding $j \uparrow V$ is definable from the names of those parameters in $V$. Finally, if $\mathbb{P}$ is mild with respect to $\kappa$ and $\kappa$ is $\lambda$ strongly compact in $V[G]$ for any $\lambda \geq \kappa$, then $\kappa$ is $\lambda$ strongly compact in $V$.

\section{The Proofs of Theorems 1, 2, 4, and 5}

We begin with the proof of Theorem 1.

Proof: We follow the proof of [1, Theorem 2]. Suppose $\lambda>\kappa$ is the least measurable cardinal and $\kappa$ is either indestructibly supercompact or indestructibly strong. Force to add a non-reflecting stationary set of ordinals of cofinality $\kappa$ to $\lambda^{+}$. After this forcing, which is $\kappa$-directed closed and $\lambda$ strategically closed (and hence is also $<\kappa$-strategically closed and $\left(\kappa^{+}, \infty\right)$-distributive), $\lambda$ remains 
the least measurable cardinal above $\kappa$. In particular, after the forcing, $\lambda$ is a measurable cardinal which is not a limit of measurable cardinals. (See [7] for a more complete discussion of the partial ordering which adds a non-reflecting stationary set of ordinals of cofinality $\gamma$ to a regular cardinal $\rho>\gamma$.$) By [18, Theorem 4.8], after the forcing, because stationary reflection for a set of ordinals of$ cofinality $\kappa<\lambda$ is violated at $\lambda^{+}, \lambda$ is not $\lambda^{+}$strongly compact. Since $\kappa$ is suitably indestructible, by reflection, $A=\left\{\delta<\kappa \mid \delta\right.$ is measurable, yet $\delta$ is neither $\delta^{+}$strongly compact nor a limit of measurable cardinals $\}$ is unbounded in $\kappa$ after the forcing has been performed. Once more, we infer by the fact adding a non-reflecting stationary set of ordinals of cofinality $\kappa$ is $\kappa$-directed closed that $A$ is unbounded in $\kappa$ in the ground model.

Having completed the proof of Theorem 1, we turn now to the proof of Theorem 2 .

Proof: Suppose $V \vDash$ "ZFC $+\kappa$ is supercompact + No cardinal $\delta>\kappa$ is measurable". Without loss of generality, by first doing a preliminary forcing if necessary, we assume in addition that $V \vDash \mathrm{GCH}$.

The partial ordering $\mathbb{P}$ used to establish Theorem 2 will be given as $\mathbb{P}^{0} * \dot{\mathbb{P}}^{1}$. To define $\mathbb{P}^{0}$, let $C=\left\{\delta<\kappa \mid \delta\right.$ is a measurable cardinal which is not $\delta^{+}$supercompact $\}$. $\mathbb{P}^{0}$ is then the reverse Easton iteration of length $\kappa$ which begins by adding a Cohen subset of $\omega$ and then adds, to every $\delta \in C$, a non-reflecting stationary set of ordinals of cofinality $\omega$. As in the proof of $[6$, Theorem 2], $\bar{V}=V^{\mathbb{P}^{0}} \vDash$ "Every measurable cardinal $\delta$ is $\delta^{+}$strongly compact $+\kappa$ is supercompact". In addition, since $\mathbb{P}^{0}$ may be defined so that $\left|\mathbb{P}^{0}\right|=\kappa$, by the results of $[17], \bar{V} \vDash$ "No cardinal $\delta>\kappa$ is measurable".

Work now in $\bar{V}$. Let $f$ be a Laver function [16] for $\kappa$, i.e., $f: \kappa \rightarrow V_{\kappa}$ is such that for every $x \in \bar{V}$ and every $\lambda \geq|\mathrm{TC}(x)|$, there is an elementary embedding $j: \bar{V} \rightarrow M$ generated by a supercompact ultrafilter over $P_{\kappa}(\lambda)$ such that $j(f)(\kappa)=x$. Our next partial ordering $\mathbb{P}^{1}$ is the reverse Easton iteration of length $\kappa$ which begins by adding a Cohen subset of $\omega$ and then (possibly) does nontrivial forcing only at cardinals $\delta<\kappa$ which are at least $\delta^{+}$supercompact in $\bar{V}$. At such a

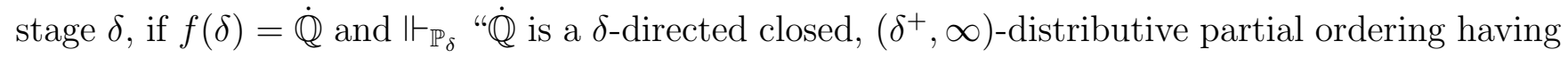


rank below the least $\bar{V}$-measurable cardinal greater than $\delta "$, then $\mathbb{P}_{\delta+1}=\mathbb{P}_{\delta} * \dot{\mathbb{Q}}$. If this is not the case, then $\mathbb{P}_{\delta+1}=\mathbb{P}_{\delta} * \dot{\mathbb{Q}}$, where $\dot{\mathbb{Q}}$ is a term for trivial forcing.

Lemma 2.1 $V^{\mathbb{P}}=\bar{V}^{\mathbb{P}^{1}} \vDash$ “ $\kappa$ 's supercompactness is indestructible under $\kappa$-directed closed, $\left(\kappa^{+}, \infty\right)$ distributive forcing".

Proof: We follow the proof of $\left[1\right.$, Lemma 2.1]. Let $\mathbb{Q} \in \bar{V}^{\mathbb{P}^{1}}$ be such that $\bar{V}^{\mathbb{P}^{1}} \vDash " \mathbb{Q}$ is $\kappa$-directed closed and $\left(\kappa^{+}, \infty\right)$-distributive". Take $\dot{\mathbb{Q}}$ as a term for $\mathbb{Q}$ such that $\Vdash_{\mathbb{P}^{1}}$ " $\dot{\mathbb{Q}}$ is $\kappa$-directed closed and $\left(\kappa^{+}, \infty\right)$-distributive". Suppose $\lambda \geq \max \left(\kappa^{+},|\mathrm{TC}(\dot{\mathbb{Q}})|\right)$ is an arbitrary cardinal, and let $\gamma=2^{\left|[\lambda]^{<\kappa}\right|}$. Take $j: \bar{V} \rightarrow M$ as an elementary embedding witnessing the $\gamma$ supercompactness of $\kappa$ generated by a supercompact ultrafilter over $P_{\kappa}(\gamma)$ such that $j(f)(\kappa)=\dot{\mathbb{Q}}$. Since $\bar{V} \vDash$ "No cardinal $\delta$ above $\kappa$ is measurable", $\gamma \geq 2^{\left[\kappa^{+}\right]^{<\kappa}}$, and $M^{\gamma} \subseteq M, M \vDash " \kappa$ is $\kappa^{+}$supercompact and no cardinal $\delta$ in the interval $(\kappa, \gamma]$ is measurable". Hence, the definition of $\mathbb{P}^{1}$ implies that $j\left(\mathbb{P}^{1} * \dot{\mathbb{Q}}\right)=\mathbb{P}^{1} * \dot{\mathbb{Q}} * \dot{\mathbb{R}} * j(\dot{\mathbb{Q}})$, where the first stage at which $\mathbb{R}$ does nontrivial forcing is well above $\gamma$. Laver's original argument from [16] now applies and shows $\bar{V}^{\mathbb{P}^{1} * \dot{\mathbb{Q}}} \vDash$ " $\kappa$ is $\lambda$ supercompact". (Simply let $G_{0} * G_{1} * G_{2}$ be $\bar{V}$-generic over $\mathbb{P}^{1} * \dot{\mathbb{Q}} * \dot{\mathbb{R}}$, lift $j$ in $\bar{V}\left[G_{0}\right]\left[G_{1}\right]\left[G_{2}\right]$ to $j: \bar{V}\left[G_{0}\right] \rightarrow M\left[G_{0}\right]\left[G_{1}\right]\left[G_{2}\right]$, take a master condition $p$ for $j^{\prime \prime} G_{1}$ and a $\bar{V}\left[G_{0}\right]\left[G_{1}\right]\left[G_{2}\right]$-generic object $G_{3}$ over $j(\mathbb{Q})$ containing $p$, lift $j$ again in $\bar{V}\left[G_{0}\right]\left[G_{1}\right]\left[G_{2}\right]\left[G_{3}\right]$ to $j: \bar{V}\left[G_{0}\right]\left[G_{1}\right] \rightarrow M\left[G_{0}\right]\left[G_{1}\right]\left[G_{2}\right]\left[G_{3}\right]$, and show by the $\gamma^{+}$-directed closure of $\mathbb{R} * j(\dot{\mathbb{Q}})$ that the supercompactness measure over $\left(P_{\kappa}(\lambda)\right)^{\bar{V}\left[G_{0}\right]\left[G_{1}\right]}$ generated by $j$ is actually a member of $\bar{V}\left[G_{0}\right]\left[G_{1}\right]$.) As $\lambda$ and $\mathbb{Q}$ were arbitrary, this completes the proof of Lemma 2.1.

Since trivial forcing is both $\kappa$-directed closed and $\left(\kappa^{+}, \infty\right)$-distributive, Lemma 2.1 implies that $\bar{V}^{\mathbb{P}^{1}}=V^{\mathbb{P}} \vDash " \kappa$ is supercompact". Also, because $\mathbb{P}^{1}$ may be defined so that $\left|\mathbb{P}^{1}\right|=\kappa$, as before, $V^{\mathbb{P}}=\bar{V}^{\mathbb{P}^{1}} \vDash$ "No cardinal $\delta>\kappa$ is measurable".

Lemma 2.2 $V^{\mathbb{P}}=\bar{V}^{\mathbb{P}^{1}} \vDash$ "Every measurable cardinal $\delta<\kappa$ is $\delta^{+}$strongly compact".

Proof: Suppose $V^{\mathbb{P}} \vDash " \delta<\kappa$ is measurable". We show that $V^{\mathbb{P}} \vDash$ " $\delta$ is $\delta^{+}$strongly compact". To do this, work in $\bar{V}$. Write $\mathbb{P}^{1}=\mathbb{P}^{\prime} * \dot{\mathbb{P}}^{\prime \prime}$, where $\left|\mathbb{P}^{\prime}\right|=\omega, \mathbb{P}^{\prime}$ is nontrivial, and $\vdash_{\mathbb{P}^{\prime}}$ " $\dot{\mathbb{P}}^{\prime \prime}$ is $\aleph_{1^{-}}$ 
strategically closed". ${ }^{3}$ By Theorem $6, \delta$ had to have been measurable in $\bar{V}$. This means by the fact $\bar{V}=V^{\mathbb{P}^{0}}$ that $\bar{V} \vDash " \delta$ is $\delta^{+}$strongly compact". If we write $\mathbb{P}^{1}=\mathbb{P}_{\delta}^{1} * \dot{\mathbb{Q}}^{*}$, by the definition of

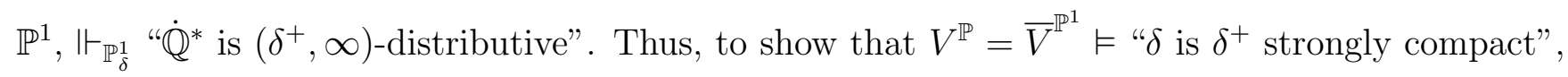
it suffices to show that $\bar{V}^{\mathbb{P}_{\delta}^{1}} \vDash " \delta$ is $\delta^{+}$strongly compact". For this, we consider two cases.

Case 1: $\bar{V} \vDash$ " $\delta$ is $\delta^{+}$supercompact". In this case, if $\left|\mathbb{P}_{\delta}^{1}\right|<\delta$, then by the results of [17], $\bar{V}^{\mathbb{P}_{\delta}^{1}} \vDash " \delta$ is $\delta^{+}$supercompact". If not, it must be true that $\left|\mathbb{P}_{\delta}^{1}\right| \geq \delta$, so by the definition of $\mathbb{P}_{\delta}^{1},\left|\mathbb{P}_{\delta}^{1}\right|=\delta$. We then let $j_{0}: \bar{V} \rightarrow M$ be an elementary embedding witnessing the $\delta^{+}$supercompactness of $\delta$ generated by a supercompact ultrafilter over $P_{\delta}\left(\delta^{+}\right)$such that $M \vDash$ " $\delta$ is not $\delta^{+}$supercompact". By the definition of $\mathbb{P}_{\delta}^{1}, j_{0}\left(\mathbb{P}_{\delta}^{1}\right)=\mathbb{P}_{\delta}^{1} * \dot{\mathbb{Q}}$, where the first ordinal at which $\mathbb{Q}$ does nontrivial forcing is well above $\delta^{+}$. Standard arguments, as given, e.g., in the proof of [8, Lemma 8.1] now show that $j_{0}$ lifts in $\bar{V}^{\mathbb{P}_{\delta}^{1}}$ to $j_{0}: \bar{V}^{\mathbb{P}_{\delta}^{1}} \rightarrow M^{j_{0}\left(\mathbb{P}_{\delta}^{1}\right)}$, i.e., $\bar{V}^{\mathbb{P}_{\delta}^{1}} \vDash " \delta$ is $\delta^{+}$supercompact". (If $G$ is $\bar{V}$-generic over $\mathbb{P}_{\delta}^{1}$, then working in $\bar{V}[G]$, we may use the usual diagonalization argument to build an $M[G]$-generic filter $H$ over $\mathbb{Q}$ with $j_{0}{ }^{\prime \prime} G \subseteq G * H .{ }^{4}$ We then have that $j_{0}$ lifts in $\bar{V}[G]$ to $j_{0}: \bar{V}[G] \rightarrow M[G][H]$.)

Case 2: $\bar{V}=V^{\mathbb{P}^{0}} \vDash " \delta$ is not $\delta^{+}$supercompact". In this case, write $\mathbb{P}^{0}=\mathbb{Q}^{\prime} * \dot{\mathbb{Q}}^{\prime \prime}$, where $\left|\mathbb{Q}^{\prime}\right|=\omega, \mathbb{Q}^{\prime}$ is nontrivial, and $\vdash_{\mathbb{Q}^{\prime}}$ "这" is $\aleph_{1}$-strategically closed". By Theorem 6 , since $\bar{V} \vDash$ " $\delta$ is measurable", $\delta$ had to have been measurable in $V$. However, by the definition of $\mathbb{P}^{0}$, since every $V$-measurable cardinal $\gamma$ which is not $\gamma^{+}$supercompact in $V$ contains a non-reflecting stationary set of ordinals of cofinality $\omega$ in $\bar{V}$, such a $\gamma$ is not weakly compact in $\bar{V}$. Consequently, it must in fact be the case that $V \vDash " \delta$ is $\delta^{+}$supercompact". Now, let $\rho$ be the least inaccessible cardinal above $\delta$ in $V$. If we write $\mathbb{P}^{0}=\mathbb{P}_{\delta}^{0} * \dot{\mathbb{Q}}^{\prime \prime \prime}$, we have that $\vdash_{\mathbb{P}_{\delta}^{0}}$ “这" is $\rho$-strategically closed and $\rho$ is the least inaccessible cardinal above $\delta "$. Thus, $\mathbb{P}_{\delta}^{1}$ is in fact definable in $V^{\mathbb{P}_{\delta}^{0}}$, and to show that $\bar{V}^{\mathbb{P}_{\delta}^{1}}=V^{\mathbb{P}^{0} * \dot{\mathbb{P}}_{\delta}^{1}} \vDash " \delta$ is $\delta^{+}$ strongly compact", it suffices to show that $V^{\mathbb{P}_{\delta}^{0} * \dot{\mathbb{P}}_{\delta}^{1}} \vDash$ " $\delta$ is $\delta^{+}$strongly compact".

For this, we use exactly the same argument as given in [5, Lemma 2.3, Claim 1 of Case 2]. We

\footnotetext{
${ }^{3}$ Although not directly relevant to this proof, it is actually true that $\Perp_{\mathbb{P}}$ " $\dot{\mathbb{P}}$ " is $\aleph_{2}$-directed closed".

${ }^{4}$ An outline of this argument is as follows. $M[G]$ remains $\delta^{+}$-closed with respect to $\bar{V}[G]$, which means that $\mathbb{Q}$ is $\prec \delta^{++}$-strategically closed in both $M[G]$ and $\bar{V}[G]$. Since $\bar{V} \vDash$ GCH and $M$ is given via an ultrapower by a supercompact ultrafilter over $P_{\delta}\left(\delta^{+}\right)$, we may let $\left\langle D_{\alpha} \mid \alpha<\delta^{++}\right\rangle \in \bar{V}[G]$ enumerate the dense open subsets of $\mathbb{Q}$ present in $M[G]$. We may then use the fact that $\mathbb{Q}$ is $\prec \delta^{++}$-strategically closed in $\bar{V}[G]$ to meet each $D_{\alpha}$ and thereby construct $H$.
} 
will not provide as many details as in [5], although we will give a reasonably complete proof. Let $j: V \rightarrow M$ be an elementary embedding witnessing the $\delta^{+}$supercompactness of $\delta$ generated by a supercompact ultrafilter over $P_{\delta}\left(\delta^{+}\right)$such that $M \vDash$ " $\delta$ is not $\delta^{+}$supercompact". Since GCH holds in both $V$ and $M$, we may let $k: M \rightarrow N$ be an elementary embedding generated by a normal measure $\mathcal{U} \in M$ having trivial Mitchell rank. It is the case that if $i: V \rightarrow N$ is an elementary embedding having critical point $\delta$ and for any $x \subseteq N$ with $|x| \leq \delta^{+}$, there is some $y \in N$ such that $x \subseteq y$ and $N \vDash "|y|<i(\delta)$ ", then $i$ witnesses the $\delta^{+}$strong compactness of $\delta$. Using this fact, it is easily verifiable that the elementary embedding $i=k \circ j$ witnesses the $\delta^{+}$strong compactness of $\delta$ in $V$. We show that this embedding lifts in $V^{\mathbb{P}_{\delta}^{0} * \dot{\mathbb{P}}_{\delta}^{1}}$ to an elementary embedding $i: V^{\mathbb{P}_{\delta}^{0} * \dot{\mathbb{P}}_{\delta}^{1}} \rightarrow N^{i\left(\mathbb{P}_{\delta}^{0} * \dot{\mathbb{P}}_{\delta}^{1}\right)}$. The lifted embedding witnesses the $\delta^{+}$strong compactness of $\delta$ in $V^{\mathbb{P}_{\delta}^{0} * \dot{\mathbb{P}}_{\delta}^{1}}$, thereby completing the proof of Lemma 2.2.

To do this, write $i\left(\mathbb{P}_{\delta}^{0} * \dot{\mathbb{P}}_{\delta}^{1}\right)=\left(\mathbb{P}_{\delta}^{0} * \dot{\mathbb{Q}}^{0} * \dot{\mathbb{R}}^{0}\right) *\left(\dot{\mathbb{P}}_{\delta}^{1} * \dot{\mathbb{Q}}^{1} * \dot{\mathbb{R}}^{1}\right)$, where for $n=0,1$, the $\dot{\mathbb{Q}}^{n}$ are terms for the portions of $i\left(\mathbb{P}_{\delta}^{0} * \dot{\mathbb{P}}_{\delta}^{1}\right)$ between $\delta$ and $k(\delta)$ and the $\dot{\mathbb{R}}^{n}$ are terms for the rest of $i\left(\mathbb{P}_{\delta}^{0} * \dot{\mathbb{P}}_{\delta}^{1}\right)$, i.e., the parts above $k(\delta)$. Note that by the definition of $\mathbb{P}_{\delta}^{0}$, since $N \vDash " \delta$ is inaccessible but is not measurable", only trivial forcing will occur at stage $\delta$ in $N$ in the definition of $i\left(\mathbb{P}_{\delta}^{0} * \dot{\mathbb{P}}_{\delta}^{1}\right)$. Thus, the ordinals at which $\mathbb{Q}^{0}$ does nontrivial forcing are composed of an unbounded subset of $N$-measurable cardinals in the interval $(\delta, k(\delta)]$. As $M \vDash$ " $\delta$ is measurable but is not $\delta^{+}$supercompact", by the definition of $\mathbb{P}_{\delta}^{0}$, nontrivial forcing occurs at stage $\delta$ in $M$ in the definition of $j\left(\mathbb{P}_{\delta}^{0}\right)$. This means that by elementarity, $\mathbb{Q}^{0}$ does nontrivial forcing at stage $k(\delta)$. Further, the ordinals at which $\mathbb{R}^{0}$ does nontrivial forcing are also composed of an unbounded subset of the $N$-measurable cardinals in the interval $(k(\delta), k(j(\delta)))$.

Let $G=G_{0} * G_{1}$ be $V$-generic over $\mathbb{P}_{\delta}^{0} * \dot{\mathbb{P}}_{\delta}^{1}$. We construct in $V\left[G_{0}\right]$ an $N\left[G_{0}\right]$-generic object $G_{1}^{0}$ over $\mathbb{Q}^{0}$ and an $N\left[G_{0}\right]\left[G_{1}^{0}\right]$-generic object $G_{2}^{0}$ over $\mathbb{R}^{0}$. We then construct in $V\left[G_{0}\right]\left[G_{1}\right]=V[G]$ an $N\left[G_{0}\right]\left[G_{1}^{0}\right]\left[G_{2}^{0}\right]\left[G_{1}\right]$-generic object $G_{1}^{1}$ over $\mathbb{Q}^{1}$ and an $N\left[G_{0}\right]\left[G_{1}^{0}\right]\left[G_{2}^{0}\right]\left[G_{1}\right]\left[G_{1}^{1}\right]$-generic object $G_{2}^{1}$ over $\mathbb{R}^{1}$. Our construction will guarantee that $i^{\prime \prime}\left(G_{0} * G_{1}\right) \subseteq\left(G_{0} * G_{1}^{0} * G_{2}^{0}\right) *\left(G_{1} * G_{1}^{1} * G_{2}^{1}\right)$. This means that $i: V \rightarrow N$ lifts to $i: V\left[G_{0}\right]\left[G_{1}\right] \rightarrow N\left[G_{0}\right]\left[G_{1}^{0}\right]\left[G_{2}^{0}\right]\left[G_{1}\right]\left[G_{1}^{1}\right]\left[G_{2}^{1}\right]$ in $V[G]=V\left[G_{0}\right]\left[G_{1}\right]$, meaning that $V^{\mathbb{P}_{\delta}^{0} * \dot{\mathbb{P}}_{\delta}^{1}} \vDash " \delta$ is $\delta^{+}$strongly compact". ${ }^{5}$

\footnotetext{
${ }^{5}$ To see that the lifted embedding satisfies the cover property mentioned above, suppose that $\gamma$ is an ordinal with
} 
To obtain $G_{1}^{0}$, note that since $k$ is generated by an ultrafilter over $\delta$ and since GCH holds in both $V$ and $N,\left|k\left(\delta^{+}\right)\right|=\left|k\left(2^{\delta}\right)\right|=\mid\left\{f \mid f: \delta \rightarrow \delta^{+}\right.$is a function $\}|=|\left[\delta^{+}\right]^{\delta} \mid=\delta^{+}$. Thus, as $N\left[G_{0}\right] \vDash "\left|\wp\left(\mathbb{Q}^{0}\right)\right|=k\left(2^{\delta}\right)$ ", we can let $\left\langle D_{\alpha} \mid \alpha<\delta^{+}\right\rangle$enumerate in $V\left[G_{0}\right]$ the dense open subsets of $\mathbb{Q}^{0}$ found in $N\left[G_{0}\right]$. Since $N\left[G_{0}\right] \vDash " \mathbb{Q}^{0}$ is $\prec \delta^{+}$-strategically closed", the argument given in Case 1 above for the construction of the generic object $H$ is applicable here as well and allows us to build $G_{1}^{0}$ in $V\left[G_{0}\right]$ which is $N\left[G_{0}\right]$-generic over $\mathbb{Q}^{0}$ in the same manner.

We next construct in $V\left[G_{0}\right]$ the desired $N\left[G_{0}\right]\left[G_{1}^{0}\right]$-generic object $G_{2}^{0}$ over $\mathbb{R}^{0}$. To do this, we first note that as $M \vDash " \delta$ is measurable but is not $\delta^{+}$supercompact", we can write $j\left(\mathbb{P}_{\delta}^{0}\right)$ as $\mathbb{P}_{\delta}^{0} * \dot{\mathbb{S}}^{0} * \dot{\mathbb{T}}^{0}$, where $\dot{\mathbb{S}}^{0}$ is a term for the partial ordering adding a non-reflecting stationary set of ordinals of cofinality $\omega$ to $\delta$, and $\dot{T}^{0}$ is a term for the rest of $j\left(\mathbb{P}_{\delta}^{0}\right)$. In addition, the ordinals at which $\mathbb{T}^{0}$ does nontrivial forcing are composed of an unbounded subset of the $M$-measurable cardinals in the interval $\left(\delta^{+}, j(\delta)\right)$. This implies that in $M, \Vdash_{\mathbb{P}_{\delta}^{0} * \dot{\mathbb{S}}^{0}}$ " $\dot{\mathbb{T}}^{0}$ is $\prec \delta^{++}$-strategically closed". Further, $\left|\left[\delta^{+}\right]^{<\delta}\right|=\delta^{+}$, and since $V \vDash \mathrm{GCH}, 2^{\delta^{+}}=\delta^{++}$. Therefore, as $j$ is generated by a supercompact ultrafilter over $P_{\delta}\left(\delta^{+}\right),\left|(j(\delta))^{+}\right|=\left|j\left(\delta^{+}\right)\right|=\left|j\left(2^{\delta}\right)\right|=\left|2^{j(\delta)}\right|=\mid\left\{f \mid f: P_{\delta}\left(\delta^{+}\right) \rightarrow \delta^{+}\right.$is a function $\}|=|\left[\delta^{+}\right]^{\delta^{+}} \mid=\delta^{++}$.

Work until otherwise specified in $M$. Consider the "term forcing" partial ordering $\mathbb{T}^{*}$ (see [10] for the first published account of term forcing or [9, Section 1.2.5, page 8]; the notion is originally due to Laver) associated with $\dot{\mathbb{T}}^{0}$, i.e., $\tau \in \mathbb{T}^{*}$ iff $\tau$ is a term in the forcing language with respect to $\mathbb{P}_{\delta}^{0} * \dot{\mathbb{S}}^{0}$ and $\vdash_{\mathbb{P}_{\delta}^{0} * \dot{\mathbb{S}}^{0}}$ " $\tau \in \dot{\mathbb{T}}^{0}$ ", ordered by $\tau \geq \sigma$ iff $\Vdash_{\mathbb{P}_{\delta}^{0} * \dot{\mathbb{S}}^{0}}$ " $\tau \geq \sigma$ ". Although $\mathbb{T}^{*}$ as defined is technically a proper class, it is possible to restrict the terms appearing in it to a sufficiently large set-sized collection, with the additional crucial property that any term $\tau$ forced to be in $\dot{\mathbb{T}}^{0}$ is also forced to be equal to an element of $\mathbb{T}^{*}$. As in [5], this can be done in such a way that $M \vDash "\left|\mathbb{T}^{*}\right|=j(\delta) "$.

Clearly, $\mathbb{T}^{*} \in M$. Also, since $\Vdash_{\mathbb{P}_{\delta}^{0} * \dot{\mathbb{S}}^{0}}$ " $\dot{\mathbb{T}}^{0}$ is $\prec \delta^{++}$-strategically closed", it can easily be verified

the property that $p \Vdash$ " $\dot{x} \subseteq \gamma$ is such that $|\dot{x}| \leq \delta^{+}$". Note that this is unambiguous, since $\mathbb{P}_{\delta}^{0} * \dot{\mathbb{P}}_{\delta}^{1}$ satsifies $\delta$-c.c. For $\alpha<\delta^{+}$, let $\dot{x}_{\alpha}$ be a term for the $\alpha^{\text {th }}$ member of $\dot{x}$. Let $y_{\alpha}=\left\{\beta \mid \exists q \geq p\left[q \Vdash " \beta=\dot{x}_{\alpha} "\right]\right\}$, and define $y^{\prime}=\bigcup_{\alpha<\delta^{+}} y_{\alpha}$. Since $\mathbb{P}_{\delta}^{0} * \dot{\mathbb{P}}_{\delta}^{1}$ satsifies $\delta$-c.c., $\left|y^{\prime}\right| \leq \delta^{+}$. By the fact $i: V \rightarrow N$ is an elementary embedding witnessing the $\delta^{+}$strong compactness of $\delta$, let $y \in N, y^{\prime} \subseteq y$ be such that $N \vDash "|y|<i(\delta)$ ". Since $N \subseteq N\left[G_{0}\right]\left[G_{1}^{0}\right]\left[G_{2}^{0}\right]\left[G_{1}\right]\left[G_{1}^{1}\right]\left[G_{2}^{1}\right]$, $y$ is the desired set covering $x$ in $N\left[G_{0}\right]\left[G_{1}^{0}\right]\left[G_{2}^{0}\right]\left[G_{1}\right]\left[G_{1}^{1}\right]\left[G_{2}^{1}\right]$. 
that $\mathbb{T}^{*}$ itself is $\prec \delta^{++}$-strategically closed in $M$ and, since $M^{\delta^{+}} \subseteq M$, in $V$ as well. Since $M \vDash$ " $2^{j(\delta)}=(j(\delta))^{+}=j\left(\delta^{+}\right)$", this means we can let $\left\langle D_{\alpha} \mid \alpha<\delta^{++}\right\rangle \in V$ enumerate the dense open subsets of $\mathbb{T}^{*}$ found in $M$ and use the same argument employed in the construction of $G_{1}^{0}$ to build in $V$ an $M$-generic object $G^{*}$ over $\mathbb{T}^{*}$.

Note that since $N$ is given by an ultrapower of $M$ via a normal ultrafilter $\mathcal{U} \in M$ over $\delta$, $[9$, Fact 2, Section 1.2.2] tells us that $k^{\prime \prime} G^{*}$ generates an $N$-generic object $G_{2}^{*}$ over $k\left(\mathbb{T}^{*}\right)$. By elementarity, $k\left(\mathbb{T}^{*}\right)$ is the term forcing in $N$ defined with respect to $k\left(j\left(\mathbb{P}_{\delta}^{0}\right)_{\delta+1}\right)=\mathbb{P}_{\delta}^{0} * \dot{\mathbb{Q}}^{0}$. Therefore, since $i\left(\mathbb{P}_{\delta}^{0}\right)=k\left(j\left(\mathbb{P}_{\delta}^{0}\right)\right)=\mathbb{P}_{\delta}^{0} * \dot{\mathbb{Q}}^{0} * \dot{\mathbb{R}}^{0}, G_{2}^{*}$ is $N$-generic over $k\left(\mathbb{T}^{*}\right)$, and $G_{0} * G_{1}^{0}$ is $k\left(\mathbb{P}_{\delta}^{0} * \dot{\mathbb{S}}^{0}\right)$-generic over $N,\left[9\right.$, Fact 1, Section 1.2.5] tells us that for $G_{2}^{0}=\left\{i_{G_{0} * G_{1}^{0}}(\tau) \mid \tau \in G_{2}^{*}\right\}, G_{2}^{0}$ is $N\left[G_{0}\right]\left[G_{1}^{0}\right]$-generic over $\mathbb{R}^{0}$.

Working in $V\left[G_{0}\right]\left[G_{1}\right]$, we build the generic objects $G_{1}^{1}$ and $G_{2}^{1}$. To construct $G_{1}^{1}$, we note that by the strategic closure properties of the partial orderings over which $G_{1}^{0}$ and $G_{2}^{0}$ are generic, $N\left[G_{0}\right]\left[G_{1}^{0}\right]\left[G_{2}^{0}\right]$ remains $\delta$-closed with respect to $V\left[G_{0}\right]\left[G_{1}^{0}\right]\left[G_{2}^{0}\right]=V\left[G_{0}\right]$. Therefore, since $\mathbb{P}_{\delta}^{1}$ is $\delta$ c.c., $N\left[G_{0}\right]\left[G_{1}^{0}\right]\left[G_{2}^{0}\right]\left[G_{1}\right]$ remains $\delta$-closed with respect to $V\left[G_{0}\right]\left[G_{1}^{0}\right]\left[G_{2}^{0}\right]\left[G_{1}\right]=V\left[G_{0}\right]\left[G_{1}\right]$. Further, since $M \vDash " \delta$ is not $\delta^{+}$supercompact", $N \vDash$ " $k(\delta)$ is not $k\left(\delta^{+}\right)$supercompact", and so any ordinals at which $\mathbb{Q}^{1}$ does nontrivial forcing lie in the interval $(\delta, k(\delta))$. This means we can construct the $N\left[G_{0}\right]\left[G_{1}^{0}\right]\left[G_{2}^{0}\right]\left[G_{1}\right]$-generic object $G_{1}^{1}$ over $\mathbb{Q}^{1}$ in $V\left[G_{0}\right]\left[G_{1}\right]$ in the same way $G_{1}^{0}$ was constructed in $V\left[G_{0}\right]$.

To build $G_{2}^{1}$, we once again work in $M$. Write $j\left(\mathbb{P}_{\delta}^{0} * \dot{\mathbb{P}}_{\delta}^{1}\right)$ as $\mathbb{P}_{\delta}^{0} * \dot{\mathbb{S}}^{0} * \dot{\mathbb{T}}^{0} * \dot{\mathbb{P}}_{\delta}^{1} * \dot{\mathbb{T}}^{1}$, where $\dot{\mathbb{S}}^{0}$ and $\dot{\mathbb{T}}^{0}$ are as before, and $\dot{\mathbb{T}}^{1}$ is a term for the portion of $j\left(\mathbb{P}_{\delta}^{0} * \dot{\mathbb{P}}_{\delta}^{1}\right)$ defined in $M$ between stages $\delta$ and $j(\delta)$. Note that since $M \vDash " \delta$ is not $\delta^{+}$supercompact", all ordinals at which $\mathbb{T}^{1}$ does nontrivial forcing lie in the interval $\left(\delta^{+}, j(\delta)\right)$.

Let $\mathbb{T}^{* *}$ be the term forcing partial ordering associated with $\mathbb{T}^{1}$, i.e., $\tau \in \mathbb{T}^{* *}$ iff $\tau$ is a term in the forcing language with respect to $\mathbb{P}_{\delta}^{0} * \dot{\mathbb{S}}^{0} * \dot{\mathbb{T}}^{0} * \dot{\mathbb{P}}_{\delta}^{1}$ and $\vdash_{\mathbb{P}_{\delta}^{0} * \dot{\mathbb{S}}^{0} * \dot{\mathbb{T}}^{0} * \dot{\mathbb{P}}_{\delta}^{1}}$ “ $\tau \in \dot{\mathbb{T}}^{1}$ ", ordered by $\tau \geq \sigma$ iff $\Vdash_{\mathbb{P}_{\delta}^{0} * \dot{\mathbb{S}}^{0} * \dot{\mathbb{T}}^{0} * \dot{\mathbb{P}}_{\delta}^{1}}$ " $\tau \geq \sigma$ ". A similar analysis to that given for the term forcing $\mathbb{T}^{*}$, using the observations made in the preceding paragraphs, now allows us to construct in $V$ an $M$-generic object $G^{* *}$ for $\mathbb{T}^{* *}$, infer that $k^{\prime \prime} G^{* *}$ generates an $N$-generic object $G_{2}^{* *}$ for the relevant term forcing partial ordering in 
$N$, and working in $V\left[G_{0}\right]\left[G_{1}\right]$, use $G_{2}^{* *}$ to create an $N\left[G_{0}\right]\left[G_{1}^{0}\right]\left[G_{2}^{0}\right]\left[G_{1}\right]\left[G_{1}^{1}\right]$-generic object $G_{2}^{1}$ over $\mathbb{R}^{1}$. Thus, in $V\left[G_{0}\right]\left[G_{1}\right], i: V \rightarrow N$ lifts to $i: V\left[G_{0}\right]\left[G_{1}\right] \rightarrow N\left[G_{0}\right]\left[G_{1}^{0}\right]\left[G_{2}^{0}\right]\left[G_{1}\right]\left[G_{1}^{1}\right]\left[G_{2}^{1}\right]$. This means that $V^{\mathbb{P}_{\delta}^{0} * \dot{\mathbb{P}}_{\delta}^{1}} \vDash " \delta$ is $\delta^{+}$strongly compact". This completes the proof of Lemma 2.2.

Lemma 2.3 $V^{\mathbb{P}}=\bar{V}^{\mathbb{P}^{1}}=V^{\mathbb{P}^{0} * \dot{\mathbb{P}}^{1}} \vDash " N o$ cardinal $\delta<\kappa$ is strongly compact".

Proof: By the definition of $\mathbb{P}^{0}, \bar{V}=V^{\mathbb{P}^{0}} \vDash$ "Unboundedly in $\kappa$ many regular cardinals $\gamma<\kappa$ contain non-reflecting stationary sets of ordinals of cofinality $\omega "$. Therefore, by [18, Theorem 4.8] and the succeeding remarks, $\bar{V}=V^{\mathbb{P}^{0}} \vDash$ "No cardinal $\delta<\kappa$ is strongly compact". Then, to show that $V^{\mathbb{P}}=\bar{V}^{\mathbb{P}^{1}} \vDash$ "No cardinal $\delta<\kappa$ is strongly compact", suppose that $V^{\mathbb{P}}=\bar{V}^{\mathbb{P}^{1}} \vDash " \delta<\kappa$ is measurable". Let $\mathbb{P}^{1}=\mathbb{P}^{\prime} * \dot{\mathbb{P}}^{\prime \prime}$ be the factorization of $\mathbb{P}^{1}$ given in Lemma 2.2. As in Lemma 2.2, by this factorization, Theorem 6 implies that $\delta$ had to have been measurable in $\bar{V}$. However, by its definition, $\mathbb{P}^{1}$ is mild with respect to $\delta$, so if $V^{\mathbb{P}}=\bar{V}^{\mathbb{P}^{1}} \vDash$ " $\delta$ is strongly compact", then $\bar{V} \vDash$ " $\delta$ is strongly compact" as well. Since we have already seen that this does not occur, $V^{\mathbb{P}}=\bar{V}^{\mathbb{P}^{1}} \vDash " \delta$ is not strongly compact". This completes the proof of Lemma 2.3.

Lemmas $2.1-2.3$ and the intervening remarks complete the proof of Theorem 2 .

Theorem 4 now follows as an immediate corollary to Theorem 2, with the model $V^{\mathbb{P}}$ constructed for Theorem 2 as its witness. To see this, it suffices to show that no strong cardinal $\delta<\kappa$ has its strongness indestructible under $\delta$-directed closed, $\left(\delta^{+}, \infty\right)$-distributive forcing. However, if this were the case, then by Theorem 1 , there would be measurable cardinals $\gamma<\delta$ which were not $\gamma^{+}$ strongly compact. By the construction of $V^{\mathbb{P}}$, this is impossible. The proof of Theorem 4 is now completed by the observation that since $\kappa$ 's supercompactness is indestructible under $\kappa$-directed closed, $\left(\kappa^{+}, \infty\right)$-distributive forcing, its strongness is as well. 
Theorem 5 follows as a corollary to the proof of Theorem 2. The definition of the partial ordering $\mathbb{P}$ used in the proof of Theorem 5 is the same as in Theorem 2, with the exception that at nontrivial stages of forcing $\delta$ in the definition of $\mathbb{P}^{1}$, we allow $\dot{\mathbb{Q}}$ to be a term for an arbitrary $\delta$-directed closed partial ordering in $V^{\mathbb{P}_{\delta}}$ having rank below the least $\bar{V}$-measurable cardinal greater than $\delta$. In other words, at a nontrivial stage of forcing $\delta$, $\dot{\mathbb{Q}}$ is such that $\vdash_{\mathbb{P}_{\delta}}$ " $\dot{\mathbb{Q}}$ is a $\delta$-directed closed partial ordering having rank below the least $\bar{V}$-measurable cardinal greater than $\delta$ ".

With this definition, the proof that $V^{\mathbb{P}} \vDash$ " $\kappa$ is indestructibly supercompact" is exactly the same as in Lemma 2.1. Further, the proof that $V^{\mathbb{P}} \vDash$ "No cardinal $\delta<\kappa$ is strongly compact" is precisely the same as in Lemma 2.3. Since just as in the proof of Theorem $2, V^{\mathbb{P}} \vDash$ "No cardinal $\delta>\kappa$ is measurable", the proof of Theorem 5 will be complete once we have shown that $V^{\mathbb{P}} \vDash$ "If $\delta<\kappa$ is a measurable cardinal which is not a limit of measurable cardinals, then $\delta$ is $\delta^{+}$strongly compact".

To do this, let $\delta<\kappa$ be such that $\bar{V} \vDash$ " $\delta$ is a measurable cardinal which is not a limit of measurable cardinals". By the definition of $\mathbb{P}^{1}$, we may write $\mathbb{P}^{1}=\mathbb{P}_{\delta}^{1} * \dot{\mathbb{Q}}^{*}$, where $\left|\mathbb{P}_{\delta}^{1}\right|<\delta$ and $\vdash_{\mathbb{P}_{\delta}^{1}}$ “这 is $\rho$-directed closed for $\rho$ the least inaccessible cardinal above $\delta$ ". Since $\bar{V} \vDash$ " $\delta$ is $\delta^{+}$strongly compact", by the results of $[17], \bar{V}^{\mathbb{P}_{\delta}^{1}} \vDash$ " $\delta$ is $\delta^{+}$strongly compact". Thus, $\bar{V}^{\mathbb{P}^{1} * \dot{\mathbb{Q}}^{*}}=\bar{V}^{\mathbb{P}^{1}}=V^{\mathbb{P}} \vDash " \delta$ is $\delta^{+}$strongly compact". We will have consequently finished the proof of Theorem 5 once we have shown that if $\bar{V}^{\mathbb{P}^{1}}=V^{\mathbb{P}} \vDash$ " $\delta$ is a measurable cardinal which is not a limit of measurable cardinals", then $\bar{V} \vDash$ " $\delta$ is a measurable cardinal which is not a limit of measurable cardinals".

For this, by the direct analogue of the factorization of $\mathbb{P}^{1}$ given in Lemma 2.2 and Theorem 6 , if $\bar{V}^{\mathbb{P}^{1}} \vDash$ " $\delta$ is a measurable cardinal", then $\bar{V} \vDash$ " $\delta$ is measurable" as well. We must therefore show that if $\bar{V}^{\mathbb{P}^{1}} \vDash$ " $\delta$ is not a limit of measurable cardinals", then $\bar{V} \vDash$ " $\delta$ is not a limit of measurable cardinals". However, if $\bar{V} \vDash$ " $\delta$ is a measurable cardinal which is a limit of measurable cardinals", then in particular, $\bar{V} \vDash$ " $\delta$ is a measurable cardinal which is a limit of measurable cardinals $\gamma$ such that $\gamma$ is not a limit of measurable cardinals". By our remarks in the preceding paragraph, such $\gamma$ are preserved to $\bar{V}^{\mathbb{P}^{1}}$, i.e., $\bar{V}^{\mathbb{P}^{1}}=V^{\mathbb{P}} \vDash " \delta$ is a measurable cardinal which is a limit of measurable cardinals". This contradiction completes the proof of Theorem 5. 


\section{The Proof of Theorem 3}

Having completed the proofs of Theorems 1, 2, 4, and 5, we turn now to the proof of Theorem 3. Proof: Suppose $V \vDash$ "ZFC $+\mathrm{GCH}+\kappa$ is the least cardinal which is both strong and $\kappa^{+}$supercompact + No cardinal $\delta>\kappa$ is measurable". As with the proof of Theorem 2, the partial ordering $\mathbb{P}$ used to establish Theorem 3 will be given as $\mathbb{P}^{0} * \dot{\mathbb{P}}^{1}$. $\mathbb{P}^{0}$ is defined as before, i.e., let $C=\left\{\delta<\kappa \mid \delta\right.$ is a measurable cardinal which is not $\delta^{+}$supercompact $\}$. $\mathbb{P}^{0}$ is then the reverse Easton iteration of length $\kappa$ which begins by adding a Cohen subset of $\omega$ and then adds, to every $\delta \in C$, a non-reflecting stationary set of ordinals of cofinality $\omega$. Once again, $\bar{V}=V^{\mathbb{P}^{0}} \vDash$ "Every measurable cardinal $\delta$ is $\delta^{+}$strongly compact".

We have now the following lemma.

Lemma $3.1 \bar{V} \vDash$ " $\kappa$ is strong".

Proof: The proof of Lemma 3.1 is very similar to the one given in [7, Lemma 2.5]. We consequently quote from this argument, making the appropriate changes where necessary. We use for the proof of this lemma the same notation and terminology as in [7, Lemma 2.5], which is taken from the introductory section of [9]. Fix $\zeta>\kappa^{+}$an arbitrarily large ordinal, and let $\lambda>\zeta$ be the least cardinal such that $\lambda=\aleph_{\lambda}$ and $\lambda$ has cofinality $\kappa$. Let $j: V \rightarrow M$ be an elementary embedding witnessing the $\lambda$ strongness of $\kappa$ generated by a $(\kappa, \lambda)$-extender of width $\kappa$ such that $M \vDash$ " $\kappa$ is not $\lambda$ strong", ${ }^{6}$ and let $i: V \rightarrow N$ be the elementary embedding witnessing the measurability of $\kappa$ generated by the normal ultrafilter $\mathcal{U}=\{x \subseteq \kappa \mid \kappa \in j(x)\}$. We then have the commutative diagram

\footnotetext{
${ }^{6}$ As in [9, page 4], an $(\alpha, \beta)$-extender $\mathcal{E}=\left\langle E_{a} \mid a \in[\beta]^{<\omega}\right\rangle$ is said to have width $\gamma$ if for all $a \in[\beta]^{<\omega}, E_{a}$ is an $\alpha$-additive ultrafilter over $[\gamma]^{|a|}$.
} 


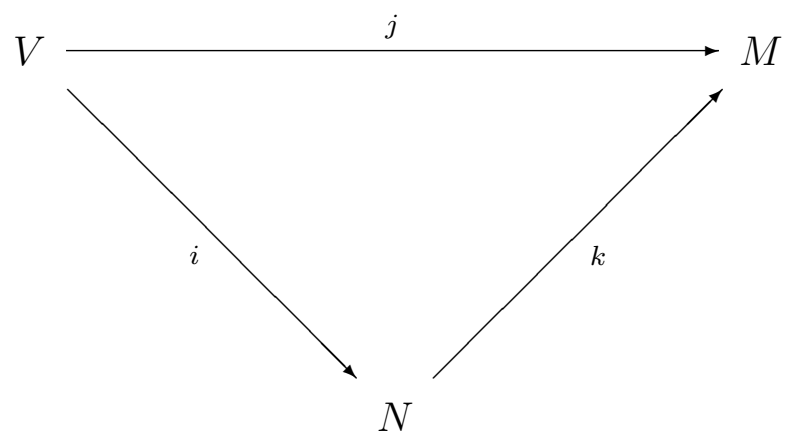

where $j=k \circ i$ and the critical point of $k$ is above $\kappa^{+}$.

Observe that $M \vDash$ "No cardinal $\rho \in(\kappa, \lambda)$ is measurable", for if this were false, then since $V_{\lambda} \subseteq M, V \vDash " \rho$ is measurable". Because $V \vDash$ "No cardinal $\delta>\kappa$ is measurable", this is impossible. As the choice of $\lambda$ implies that $M \vDash " \lambda$ is singular", this means that in $M$, the least measurable cardinal $\delta>\kappa$ is such that $\delta>\lambda$.

For any ordinal $\alpha$, define $\sigma_{\alpha}$ as the least ordinal greater than $\alpha$ such that $\alpha$ is not $\sigma_{\alpha}$ strong if such an ordinal exists, and $\sigma_{\alpha}=0$ otherwise. Define $f: \kappa \rightarrow \kappa$ as $f(\alpha)=$ The least inaccessible cardinal greater than $\sigma_{\alpha}$. By our choice of $\lambda$ and the preceding paragraph, $\kappa<\lambda<j(f)(\kappa)<\delta$, where $\delta$ is the least measurable cardinal in $M$ greater than $\kappa$, i.e., the least element above $\kappa$ to which $j\left(\mathbb{P}^{0}\right)$ adds a non-reflecting stationary set of ordinals of cofinality $\omega$.

Note now that $M=\left\{j(g)(a) \mid a \in[\lambda]^{<\omega}, \operatorname{dom}(g)=[\kappa]^{|a|}, g:[\kappa]^{|a|} \rightarrow V\right\}=\{k(i(g))(a) \mid a \in$ $\left.[\lambda]^{<\omega}, \operatorname{dom}(g)=[\kappa]^{|a|}, g:[\kappa]^{|a|} \rightarrow V\right\}$. By defining $\gamma=i(f)(\kappa)$, we have $k(\gamma)=k(i(f)(\kappa))=$ $j(f)(\kappa)>\lambda$. This means $j(g)(a)=k(i(g))(a)=k\left(i(g) \uparrow[\gamma]^{|a|}\right)(a)$ for $a \in[\lambda]^{<\omega}$. Hence, $M=$ $\left\{k(h)(a) \mid a \in[\lambda]^{<\omega}, h \in N, \operatorname{dom}(h)=[\gamma]^{|a|}, h:[\gamma]^{|a|} \rightarrow N\right\}$. By elementarity, we must have $N \vDash " \kappa$ is not strong and $\kappa<\gamma=i(f)(\kappa)<\delta_{0}=$ The least measurable cardinal in $N$ greater than $\kappa=$ The least element above $\kappa$ to which $i\left(\mathbb{P}^{0}\right)$ adds a non-reflecting stationary set of ordinals of cofinality $\omega "$, since $M \vDash " k(\kappa)=\kappa$ is not strong and $k(\kappa)=\kappa<k(\gamma)=k(i(f)(\kappa))=j(f)(\kappa)<$ $k\left(\delta_{0}\right)=\delta$ ". Thus, $k$ can be assumed to be generated by an $N$-extender of width $\gamma \in\left(\kappa, \delta_{0}\right)$.

Write $i\left(\mathbb{P}^{0}\right)=\mathbb{P}^{0} * \dot{\mathbb{Q}}^{0}$, where $\dot{\mathbb{Q}}^{0}$ is a term for the portion of $i\left(\mathbb{P}^{0}\right)$ which does nontrivial forcing on ordinals in the interval $[\kappa, i(\kappa))$. By elementarity and the choice of $\lambda, N \vDash " \kappa$ is $\kappa^{+}$supercompact", since $M \vDash " k(\kappa)=\kappa$ is $k\left(\kappa^{+}\right)=\kappa^{+}$supercompact". Therefore, the ordinals on which $\mathbb{Q}^{0}$ does nontrivial forcing actually lie in the interval $(\kappa, i(\kappa))$, or more precisely, in the interval $\left[\delta_{0}, i(\kappa)\right)$. 
This means that if $G_{0}$ is $V$-generic over $\mathbb{P}^{0}$, the argument from Lemma 2.2 for the construction of the generic object $G_{1}^{0}$ can be applied here as well to construct in $V\left[G_{0}\right]$ an $N\left[G_{0}\right]$-generic object $G_{1}^{*}$ over $\mathbb{Q}^{0}$. Since $i^{\prime \prime} G_{0} \subseteq G_{0} * G_{1}^{*}, i$ lifts in $V\left[G_{0}\right]$ to $i: V\left[G_{0}\right] \rightarrow N\left[G_{0}\right]\left[G_{1}^{*}\right]$, and since $k^{\prime \prime} G_{0}=G_{0}$ and $k(\kappa)=\kappa, k$ lifts in $V\left[G_{0}\right]$ to $k: N\left[G_{0}\right] \rightarrow M\left[G_{0}\right]$. By [9, Fact 3, Section 1.2.2], $k: N\left[G_{0}\right] \rightarrow M\left[G_{0}\right]$ can also be assumed to be generated by an extender of width $\gamma \in\left(\kappa, \delta_{0}\right)$.

In analogy to the preceding paragraph, write $j\left(\mathbb{P}^{0}\right)=\mathbb{P}^{0} * \dot{\mathbb{Q}}^{1}$. By the last sentence of the preceding paragraph and the fact $\delta_{0}$ is the least ordinal at which $\mathbb{Q}^{0}$ does nontrivial forcing, we can use [9, Fact 2, Section 1.2.2] to infer that $H=\left\{p \in \mathbb{Q}^{1} \mid \exists q \in k^{\prime \prime} G_{1}^{*}[q \geq p]\right\}$ is $M\left[G_{0}\right]$-generic over $k\left(\mathbb{Q}^{0}\right)=\mathbb{Q}^{1}$. Thus, $k$ lifts to $k: N\left[G_{0}\right]\left[G_{1}^{*}\right] \rightarrow M\left[G_{0}\right][H]$, and we get the new commutative diagram

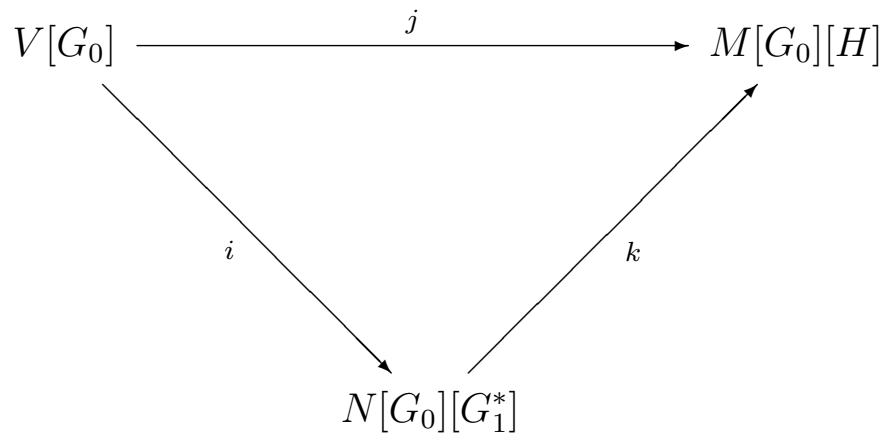

Since $M \vDash$ "No cardinal $\rho \in(\kappa, \lambda]$ is measurable", the ordinals at which $\mathbb{Q}^{1}$ does nontrivial forcing lie in the interval $(\lambda, j(\kappa))$. Thus, as $V_{\lambda} \subseteq M, V_{\lambda}\left[G_{0}\right] \subseteq M\left[G_{0}\right]$, and as $\mathbb{Q}^{1}$ is only defined on ordinals in the interval $(\lambda, j(\kappa)), V_{\lambda}\left[G_{0}\right]$ is the set of all sets of rank less than $\lambda$ in $M\left[G_{0}\right][H]$. Hence, $j$ is a $\lambda$ strongness embedding. Since both $\zeta$ and $\lambda$ may be chosen arbitrarily large, this completes the proof of Lemma 3.1.

Work now in $\bar{V}$. Before defining $\mathbb{P}$, we recall a definition from [14]. If $\mathcal{A}$ is a collection of partial orderings, then the lottery sum is the partial ordering $\oplus \mathcal{A}=\{\langle\mathbb{P}, p\rangle \mid \mathbb{P} \in \mathcal{A}$ and $p \in \mathbb{P}\} \cup\{0\}$, ordered with 0 below everything and $\langle\mathbb{P}, p\rangle \leq\left\langle\mathbb{P}^{\prime}, p^{\prime}\right\rangle$ iff $\mathbb{P}=\mathbb{P}^{\prime}$ and $p \leq p^{\prime}$. Intuitively, if $G$ is $V$-generic over $\oplus \mathcal{A}$, then $G$ first selects an element of $\mathcal{A}$ (or as Hamkins says in [14], "holds a lottery among the posets in $\mathcal{A}$ ") and then forces with it. The terminology "lottery sum" is due to 
Hamkins, although the concept of the lottery sum of partial orderings has been around for quite some time and has been referred to at different junctures via the expressions "disjoint sum of partial orderings," "side-by-side forcing," and "choosing which partial ordering to force with generically."

We complete the definition of $\mathbb{P}$ by defining $\mathbb{P}^{1}$ as the reverse Easton iteration of length $\kappa$ which begins by adding a Cohen subset of $\omega$ and then (possibly) does nontrivial forcing only at cardinals $\delta<\kappa$ which are measurable in $\bar{V}$. If $\bar{V} \vDash$ " $\delta$ is $\delta^{+}$supercompact", then $\mathbb{P}_{\delta+1}=\mathbb{P}_{\delta} * \dot{\mathbb{Q}}_{\delta}$, where $\dot{\mathbb{Q}}_{\delta}$ is a term for the partial ordering adding a non-reflecting stationary set of ordinals of cofinality $\omega$ to $\delta$. If $\bar{V} \vDash$ " $\delta$ is not $\delta^{+}$supercompact", then $\mathbb{P}_{\delta+1}=\mathbb{P}_{\delta} * \dot{\mathbb{Q}}_{\delta}$, where $\dot{\mathbb{Q}}_{\delta}$ is a term for the lottery sum of all $<\delta$-strategically closed, $\left(\delta^{+}, \infty\right)$-distributive partial orderings in $\bar{V}^{\mathbb{P}_{\delta}}$ which have rank below the least $\bar{V}$-measurable cardinal greater than $\delta$. We explicitly note that the definition of $\mathbb{P}^{1}$ uses a lottery sum, instead of a Laver function as earlier, in order to emphasize the similarities between the proofs of Lemma 3.4 and [14, Theorem 4.10].

Lemma $3.2 V^{\mathbb{P}} \vDash$ "No cardinal $\delta<\kappa$ is strong".

Proof: We show in turn that $V^{\mathbb{P}^{0}}=\bar{V} \vDash$ "No cardinal $\delta<\kappa$ is strong" and $V^{\mathbb{P}}=\bar{V}^{\mathbb{P}^{1}}=V^{\mathbb{P}^{0}} * \dot{\mathbb{P}}^{1} \vDash$ "No cardinal $\delta<\kappa$ is strong". By the definition of $\mathbb{P}^{0}$ and the fact that $V \vDash$ " $\kappa$ is the least cardinal which is both strong and $\kappa^{+}$supercompact", $\bar{V} \vDash$ "Any cardinal $\delta<\kappa$ which was strong in $V$ is non-measurable, since $\delta$ was not $\delta^{+}$supercompact and therefore contains a non-reflecting stationary set of ordinals of cofinality $\omega^{\prime \prime}$. Write $\mathbb{P}^{0}=\mathbb{Q}^{\prime} * \dot{\mathbb{Q}}^{\prime \prime}$, where $\left|\mathbb{Q}^{\prime}\right|=\omega, \mathbb{Q}^{\prime}$ is nontrivial, and $\Vdash_{\mathbb{Q}^{\prime}}$ " $\dot{\mathbb{Q}}^{\prime \prime}$ is $\aleph_{1}$-strategically closed". By Theorem 6 , if $\bar{V} \vDash " \delta$ is strong", then $V \vDash$ " $\delta$ is strong" as well. Since we have just seen that this cannot be the case if $\delta<\kappa, V^{\mathbb{P}^{0}}=\bar{V} \vDash$ "No cardinal $\delta<\kappa$ is strong". However, by writing $\mathbb{P}^{1}=\mathbb{P}^{\prime} * \dot{\mathbb{P}}^{\prime \prime}$, where $\left|\mathbb{P}^{\prime}\right|=\omega, \mathbb{P}^{\prime}$ is nontrivial, and $\vdash_{\mathbb{P}^{\prime}}$ " $\dot{\mathbb{P}}^{\prime \prime}$ is $\aleph_{1}$-strategically closed", Theorem 6 once again implies that if $\bar{V}^{\mathbb{P}^{1}} \vDash$ " $\delta$ is strong", then $\bar{V} \vDash$ " $\delta$ is strong" as well. Consequently, since $\bar{V} \vDash$ "No cardinal $\delta<\kappa$ is strong", $V^{\mathbb{P}}=\bar{V}^{\mathbb{P}^{1}}=V^{\mathbb{P}^{0} * \dot{\mathbb{P}}^{1}} \vDash$ "No cardinal $\delta<\kappa$ is strong". This completes the proof of Lemma 3.2.

Lemma 3.3 $V^{\mathbb{P}}=\bar{V}^{\mathbb{P}^{1}} \vDash$ "Every measurable cardinal $\delta \leq \kappa$ is $\delta^{+}$strongly compact". 
Proof: By the definition of $\mathbb{P}^{1}$, if $\delta<\kappa$ and $\bar{V} \vDash " \delta$ is $\delta^{+}$supercompact", then $V^{\mathbb{P}}=\bar{V}^{\mathbb{P}^{1}} \vDash " \delta$ contains a non-reflecting stationary set of ordinals of cofinality $\omega$ and hence is non-measurable". For $\delta<\kappa$, this will consequently allow us to use virtually the same arguments as in the proof of Case 2 of Lemma 2.2. We therefore indicate the only two places where the proofs are slightly different, and the necessary modifications that need to be made, using the same notation as in Lemma 2.2. These two changes occur in the construction of the generic objects $G_{1}^{1}$ and $G_{2}^{1}$ in Case 2 , because of the use of lottery sums in the definition of $\mathbb{P}^{1}$. However, by forcing if necessary above a condition opting for trivial forcing at stage $\delta$ in $M^{\mathbb{P}_{\delta}^{0} * \dot{\mathbb{S}}^{0} * \dot{\mathbb{T}}^{0} * \dot{\mathbb{P}}_{\delta}^{1}}$, we will have that $\mathbb{T}^{1}$ is forcing equivalent to a

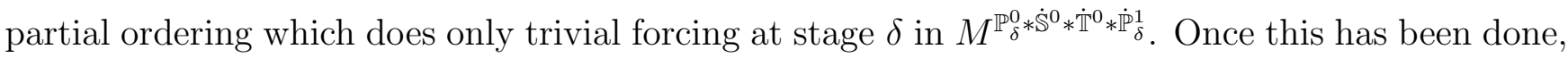

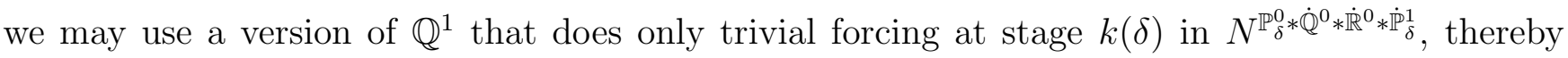
allowing us to construct $G_{1}^{1}$ as before. $G_{2}^{1}$ is also built as earlier, using the fact that the ordinals at which $\mathbb{T}^{1}$ does nontrivial forcing lie in the interval $\left(\delta^{+}, j(\delta)\right)$. Thus, Lemma 3.3 is true if $\delta<\kappa$.

For $\delta=\kappa$, we note that $\bar{V} \vDash$ " $\kappa$ is not $2^{\kappa}=\kappa^{+}$supercompact". This is since [7, Lemma 2.1] tells us that any cardinal $\delta$ which is both $2^{\delta}$ supercompact and strong must be a limit of strong cardinals. As this is false by Lemma $3.2, \bar{V} \vDash$ " $\kappa$ is $\kappa^{+}$strongly compact but is not $\kappa^{+}$supercompact". The arguments of Case 2 of Lemma 2.2 as modified in the preceding paragraph are thus applicable and show that $V^{\mathbb{P}}=\bar{V}^{\mathbb{P}^{1}} \vDash$ " $\kappa$ is $\kappa^{+}$strongly compact". This completes the proof of Lemma 3.3.

Lemma 3.4 $V^{\mathbb{P}}=\bar{V}^{\mathbb{P}^{1}} \vDash$ " $\kappa$ 's strongness is indestructible under $<\kappa$-strategically closed, $\left(\kappa^{+}, \infty\right)$ distributive forcing".

Proof: Suppose $\mathbb{Q} \in \bar{V}^{\mathbb{P}^{1}}$ is such that $\bar{V}^{\mathbb{P}^{1}} \vDash " \mathbb{Q}$ is $<\kappa$-strategically closed and $\left(\kappa^{+}, \infty\right)$-distributive".

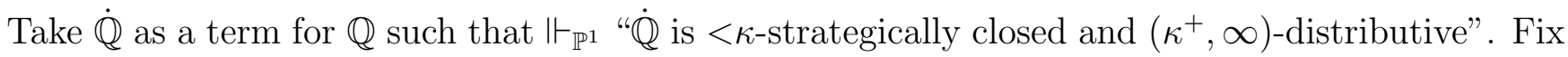
$\zeta>\kappa^{+}$an arbitrarily large ordinal, and assume that $\lambda>\max (\zeta,|\mathrm{TC}(\dot{\mathbb{Q}})|)$ is the least cardinal of cofinality $\kappa$ such that $\lambda=\aleph_{\lambda}$. Let $j: \bar{V} \rightarrow M$ be an elementary embedding witnessing the $\lambda$ strongness of $\kappa$ generated by a $(\kappa, \lambda)$-extender of width $\kappa$.

We now show that the embedding $j$ lifts in $\bar{V}^{\mathbb{P} * \dot{\mathbb{Q}}}$ to $j: \bar{V}^{\mathbb{P} * \dot{\mathbb{Q}}} \rightarrow M^{j(\mathbb{P} * \dot{\mathbb{Q}})}$. The methods for doing this are quite similar to those given in the proof of [14, Theorem 4.10] (as well as elsewhere). For 
the benefit of readers, we give the argument here as well, taking the liberty to quote freely from [14, Theorem 4.10]. Because $j$ is an extender embedding, we have that $M=\left\{j(f)(a) \mid a \in[\lambda]^{<\omega}\right.$, $f \in \bar{V}$, and $\left.\operatorname{dom}(f)=[\kappa]^{|a|}\right\}$. Since $\bar{V} \vDash " \kappa$ is not $\kappa^{+}$supercompact" and $V_{\lambda} \subseteq M$ (which means that $M \vDash$ "There are no measurable cardinals in the interval $(\kappa, \lambda]$ "), by forcing above the appropriate condition, we will have that $j(\mathbb{P})$ is forcing equivalent to $\mathbb{P} * \dot{\mathbb{Q}} * \dot{\mathbb{R}}$. Consequently, we may infer that the first ordinal at which $\mathbb{R}$ does a lottery sum is above $\lambda$. Since $\lambda$ has been chosen to have cofinality $\kappa$, we may assume that $M^{\kappa} \subseteq M$. This means that if $G$ is $\bar{V}$-generic over $\mathbb{P}$ and $H$ is $\bar{V}[G]$-generic over $\mathbb{Q}, \mathbb{R}$ is $\prec \kappa^{+}$-strategically closed in both $\bar{V}[G][H]$ and $M[G][H]$, and $\mathbb{R}$ is $\lambda$-strategically closed in $M[G][H]$.

As in [14], by using a suitable coding that allows us to identify finite subsets of $\lambda$ with elements of $\lambda$, by the definition of $M$, there must be some $\alpha_{0}<\lambda$ and function $g$ such that $\dot{\mathbb{Q}}=j(g)\left(\alpha_{0}\right)$. Let $N=\left\{i_{G * H}(\dot{z}) \mid \dot{z}=j(f)\left(\kappa, \alpha_{0}, \lambda\right)\right.$ for some function $\left.f \in \bar{V}\right\}$. It is easy to verify that $N \prec M[G][H]$, that $N$ is closed under $\kappa$ sequences in $\bar{V}[G][H]$, and that $\kappa, \alpha_{0}, \lambda, \mathbb{Q}$, and $\mathbb{R}$ are all elements of $N$. Further, since $\mathbb{R}$ is $j(\kappa)$-c.c. in $M[G][H]$ and there are only $2^{\kappa}=\kappa^{+}$many functions $f: \kappa \rightarrow V_{\kappa}$ in $\bar{V}$, there are at most $\kappa^{+}$many dense open subsets of $\mathbb{R}$ in $N$. Therefore, since $\mathbb{R}$ is $\prec \kappa^{+}$-strategically closed in both $M[G][H]$ and $\bar{V}[G][H]$, we can use the argument for the construction of the generic object $G_{1}^{0}$ given in Lemma 2.2 to build $H^{\prime} \subseteq \mathbb{R}$ in $\bar{V}[G][H]$ which is $N$-generic.

We show now that $H^{\prime}$ is actually $M[G][H]$-generic over $\mathbb{R}$. If $D$ is a dense open subset of $\mathbb{R}$ in $M[G][H]$, then $D=i_{G * H}(\dot{D})$ for some name $\dot{D} \in M$. Consequently, $\dot{D}=j(f)\left(\kappa, \kappa_{1}, \ldots, \kappa_{n}\right)$ for some function $f \in \bar{V}$ and $\kappa<\kappa_{1}<\cdots<\kappa_{n}<\lambda$. Let $\bar{D}$ be a name for the intersection of all $i_{G * H}\left(j(f)\left(\kappa, \alpha_{1}, \ldots, \alpha_{n}\right)\right)$, where $\kappa<\alpha_{1}<\cdots<\alpha_{n}<\lambda$ is such that $j(f)\left(\kappa, \alpha_{1}, \ldots, \alpha_{n}\right)$ yields a name for a dense open subset of $\mathbb{R}$. Since this name can be given in $M$ and $\mathbb{R}$ is $\lambda$-strategically closed in $M[G][H]$ and therefore $(\lambda, \infty)$-distributive in $M[G][H], \bar{D}$ is a name for a dense open subset of $\mathbb{R}$ which is definable without the parameters $\kappa_{1}, \ldots, \kappa_{n}$. Hence, by its definition, $i_{G * H}(\bar{D}) \in N$. Thus, since $H^{\prime}$ meets every dense open subset of $\mathbb{R}$ present in $N, H^{\prime} \cap i_{G * H}(\bar{D}) \neq \emptyset$, so since $\bar{D}$ is forced to be a subset of $\dot{D}, H^{\prime} \cap i_{G * H}(\dot{D}) \neq \emptyset$. This means $H^{\prime}$ is $M[G][H]$-generic over $\mathbb{R}$, so in $\bar{V}[G][H]$, as $j^{\prime \prime} G \subseteq G * H * H^{\prime}, j$ lifts to $j: \bar{V}[G] \rightarrow M[G][H]\left[H^{\prime}\right]$ via the definition $j\left(i_{G}(\tau)\right)=i_{G * H * H^{\prime}}(j(\tau))$. 
It remains to lift $j$ through the forcing $\mathbb{Q}$ while working in $\bar{V}[G][H]$. To do this, it suffices to show that $j^{\prime \prime} H \subseteq j(\mathbb{Q})$ generates an $M[G][H]\left[H^{\prime}\right]$-generic object $H^{\prime \prime}$ over $j(\mathbb{Q})$. Given a dense open subset $D \subseteq j(\mathbb{Q}), D \in M[G][H]\left[H^{\prime}\right], D=i_{G * H * H^{\prime}}(\dot{D})$ for some name $\dot{D}=j(\vec{D})(a)$, where $a \in[\lambda]^{<\omega}$ and $\vec{D}=\left\langle D_{\sigma} \mid \sigma \in[\kappa]^{|a|}\right\rangle$ is a function. We may assume that every $D_{\sigma}$ is a dense open subset of $\mathbb{Q}$. Since $\mathbb{Q}$ is $\left(\kappa^{+}, \infty\right)$-distributive (and hence is of course also $(\kappa, \infty)$-distributive), it follows that $D^{\prime}=\bigcap_{\sigma \in[\kappa]^{|a|}} D_{\sigma}$ is a dense open subset of $\mathbb{Q}$. As $j\left(D^{\prime}\right) \subseteq D$ and $H \cap D^{\prime} \neq \emptyset$, $j^{\prime \prime} H \cap D \neq \emptyset$. Thus, $H^{\prime \prime}=\left\{p \in j(\mathbb{Q}) \mid \exists q \in j^{\prime \prime} H[q \geq p]\right\}$ is our desired generic object, and $j$ lifts in $\bar{V}[G][H]$ to $j: \bar{V}[G][H] \rightarrow M[G][H]\left[H^{\prime}\right]\left[H^{\prime \prime}\right]$. This final lifted version of $j$ is $\lambda$ strong since $V_{\lambda} \subseteq M$, meaning $\left(V_{\lambda}\right)^{\bar{V}[G][H]} \subseteq M[G][H] \subseteq M[G][H]\left[H^{\prime}\right]\left[H^{\prime \prime}\right]$. Since both $\zeta$ and $\lambda$ can be made arbitrarily large, this completes the proof of Lemma 3.4.

Since trivial forcing is both $<\kappa$-strategically closed and $\left(\kappa^{+}, \infty\right)$-distributive, Lemma 3.4 implies that $\bar{V}^{\mathbb{P}^{1}}=V^{\mathbb{P}} \vDash " \kappa$ is strong". Also, because $\mathbb{P}$ may be defined so that $|\mathbb{P}|=\kappa$, as before, $V^{\mathbb{P}} \vDash$ "No cardinal $\delta>\kappa$ is measurable". In addition, Lemma 3.2 and the argument found in the last paragraph of Lemma 3.3 imply that $V^{\mathbb{P}}=\bar{V}^{\mathbb{P}^{1}} \vDash " \kappa$ is not $\kappa^{+}$supercompact".

Lemma 3.5 $\bar{V}^{\mathbb{P}^{1}}=V^{\mathbb{P}} \vDash$ "If $\delta$ is measurable, then $\delta$ is not $\delta^{+}$supercompact".

Proof: Suppose $\bar{V}^{\mathbb{P}^{1}}=V^{\mathbb{P}} \vDash$ " $\delta$ is $\delta^{+}$supercompact". By our remarks in the paragraph immediately following the proof of Lemma 3.4, we may assume without loss of generality that $\delta<\kappa$. If we then use the factorization of $\mathbb{P}^{1}=\mathbb{P}^{\prime} * \dot{\mathbb{P}}^{\prime \prime}$ given in Lemma 3.2, we may infer by Theorem 6 that since $\bar{V}^{\mathbb{P}^{1}}=V^{\mathbb{P}} \vDash$ " $\delta$ is $\delta^{+}$supercompact", it follows that $\bar{V} \vDash$ " $\delta$ is $\delta^{+}$supercompact" as well. However, as we have already observed in the proof of Lemma 3.3, by the definition of $\mathbb{P}^{1}$, if $\delta<\kappa$ and $\bar{V} \vDash$ " $\delta$ is $\delta^{+}$supercompact", then $V^{\mathbb{P}}=\bar{V}^{\mathbb{P}^{1}} \vDash$ " $\delta$ contains a non-reflecting stationary set of ordinals of cofinality $\omega$ and hence is non-measurable". This contradiction completes the proof of Lemma 3.5.

By Lemmas 3.5, 3.3, and the remarks after the proof of Lemma 3.4, $V^{\mathbb{P}} \vDash$ "Level by level 
inequivalence holds". This observation, together with Lemmas $3.1-3.4$ and the intervening comments, complete the proof of Theorem 3.

Except for the fact that the proofs of Lemmas 2.2 and 3.3 require $\left(\delta^{+}, \infty\right)$-distributivity at each nontrivial stage of forcing $\delta$ in the definition of the relevant partial ordering $\mathbb{P}^{1}$, there is no reason prima facie to believe that the amount of indestructibility forced must include this additional condition. We therefore ask if this restriction can be removed. In addition, is it possible to prove an analogue of Theorem 2 in which level by level inequivalence between strong compactness and supercompactness holds? Further, is it possible to prove an analogue of Theorem 4 for a universe with no restrictions on its large cardinal structure? Finally, is it possible to prove analogues of Theorems 2, 3, and 5 in which $\kappa$ is neither the least strongly compact nor least strong cardinal? These are the questions with which we conclude this paper.

\section{References}

[1] A. Apter, "Indestructibility and Level by Level Equivalence and Inequivalence", Mathematical Logic Quarterly 53, 2007, 78-85.

[2] A. Apter, "Indestructibility and Measurable Cardinals with Few and Many Measures", Archive for Mathematical Logic 47, 2008, 101-110.

[3] A. Apter, "Indestructibility and Stationary Reflection", Mathematical Logic Quarterly 55, 2009, 228-236.

[4] A. Apter, "Indestructibility, Strongness, and Level by Level Equivalence", Fundamenta Mathematicae 177, 2003, 45-54.

[5] A. Apter, "On Level by Level Equivalence and Inequivalence between Strong Compactness and Supercompactness", Fundamenta Mathematicae 171, 2002, 77-92. 
[6] A. Apter, "Strong Compactness, Measurability, and the Class of Supercompact Cardinals", Fundamenta Mathematicae 16\%, 2001, 65-78.

[7] A. Apter, J. Cummings, "Identity Crises and Strong Compactness II: Strong Cardinals", Archive for Mathematical Logic 40, 2001, 25-38.

[8] A. Apter, J. D. Hamkins, "Indestructibility and the Level-by-Level Agreement between Strong Compactness and Supercompactness", Journal of Symbolic Logic 67, 2002, 820-840.

[9] J. Cummings, "A Model in which GCH Holds at Successors but Fails at Limits", Transactions of the American Mathematical Society 329, 1992, 1-39.

[10] M. Foreman, "More Saturated Ideals", in: Cabal Seminar 79-81, Lecture Notes in Mathematics 1019, Springer-Verlag, Berlin and New York, 1983, 1-27.

[11] M. Gitik, S. Shelah, "On Certain Indestructibility of Strong Cardinals and a Question of Hajnal", Archive for Mathematical Logic 28, 1989, 35-42.

[12] J. D. Hamkins, "Gap Forcing", Israel Journal of Mathematics 125, 2001, 237-252.

[13] J. D. Hamkins, "Gap Forcing: Generalizing the Lévy-Solovay Theorem", Bulletin of Symbolic Logic 5, 1999, 264-272.

[14] J. D. Hamkins, "The Lottery Preparation", Annals of Pure and Applied Logic 101, 2000, $103-146$.

[15] T. Jech, Set Theory: The Third Millennium Edition, Revised and Expanded, Springer-Verlag, Berlin and New York, 2003.

[16] R. Laver, "Making the Supercompactness of $\kappa$ Indestructible under $\kappa$-Directed Closed Forcing", Israel Journal of Mathematics 29, 1978, 385-388.

[17] A. Lévy, R. Solovay, "Measurable Cardinals and the Continuum Hypothesis", Israel Journal of Mathematics 5, 1967, 234-248. 
[18] R. Solovay, W. Reinhardt, A. Kanamori, "Strong Axioms of Infinity and Elementary Embeddings", Annals of Mathematical Logic 13, 1978, 73-116. 